\title{
NUMERICAL SIMULATION OF A VISCOUS VORTEX RING INTERACTION WITH A DENSITY INTERFACE $\dagger$
}

\author{
Daniel L. Marcus and John B. Bell \\ Lawrence Livermore National Laboratory \\ Livermore, CA 94550
}

EXTENDED ABSTRACT

\section{Introduction}

When a vortex-dominated flow interacts with a sharp density interface, the dynamics are characterized by the interaction of baroclinically generated vorticity with the already existing vorticity field. This can be scen in many natural and technological settings; examples are the interaction of a ship or submarine wake with a thermocline, the collision of a buoyant thermal with a temperature inversion, and the interaction of a vortex flow with a flame front [1]. This problem also serves as a generic model for turbulent mixing and entrainment processes across sharp demsity interfaces [2].

The interaction between vortices and a free surface, which corresponds to the case where the density jump is very large, has been studied fairly extensively, both experimentally [3] and computationally $[4,5,6,7]$. By comparison, the literature for the more general case of vortex pairs and rings interacting with sharp density interfaces is relatively sparse. Experiments and numerical studies have been performed $[1,2]$, but the numerical șimulations were confined primarily to vortex pairs, restricted

\footnotetext{
† This work was performed under the auspices of the U.S. Department of Energy by the Lawrence Livermore National Laboratory under contract No. W-7405-Eng-48. Partial support was provided by the Applied Mathematical Sciences Program of the Office of Energy Rescarch under contract No. W-7405- Eng-48 and by the Defense Nuclear Agency under IACRO 90.824 .
} 
to the inviscid case, and the effect of density variation modeled under the Boussinesq approximation. The experiments were also confined to the Boussinesq regime. In this paper, we study the motion of a vortex ring in a sharply stratified, viscous fluid via a numerical solution of the full Navier-Stokes equations with finite-amplitude dersity variation. Both Boussinesq and non-Boussinesq flow regimes will be studied, the effect of viscosity on the interaction will be examined, and three-dimensional aspects of the motion will be addressed, such as Widnall instability of the vortex ring and vortex reconnection at the interface.

\section{Numerical method}

The method [8] is a generalization to variable density flows of the second-order projection method for the incompressible Navier-Stokes equations developed by Bell et al. [9], itself a higher-order extension of Chorin's projection algorithm [10], based on a discrete form of the Hodge decomposition, which states that a vector field can be decomposed into the gradient of a scalar potential and a divergence-free component.

In its most basic form, the projection method requires the solution of advection-diffusion equations, which are then projected onto the space of divergence-free vector fields. The projection is accomplished via a discrete-Galerkin finite difference formulation using a local basis for discretely divergence-free vector fields developed by Stephens et al. [11] and Solomon and Szymczak [12] for the steady Navier-Stokes equations. The gencralization to variable density flow is made by expressing the standard projection with respect to a density weighted inner product. The algorithm is extended to higher order by introducing more coupling between the advection-diffusion step and the projection, and by incorporating a variant of the unsplit second-order Godunov methodology developed by Colella [13] for inviscid, compressible flows into the evaluation of the nonlinear advection terms in the momentum equations. This provides a temperal discretization that is second-order for smooth flows and stable in regions with steep gradients, even for singular initial data and in the limit of vanishing viscosity. These properties make the method extremely well suited for application to flows with baroclinic vorticity generation; the evolving interface is simply captured in the course of solving the mass conservation equation. 


\section{Current Results}

In the viscous interaction of a vortex ring with a density interface, if surface tension along the interface is neglected, the flow is governed by three dimensionless parameters. The Atwood number represents the magnitude of the density jump across the interface, and is given by $A=\frac{\rho_{1}-\rho_{2}}{\rho_{1}+\rho_{2}}$. The inverse Froude number governs the relative importance of inertial and gravitational effects. It is defined as $F^{-2}=\frac{a^{3} g}{\Gamma^{2}}$, where $a$ is the diameter of the vortex ring, $\Gamma$ its circulation, and $g$ is the gravitational acceleration. The Reynolds number provides the familiar scaling of inertial to viscous effects, and here is based on the strength of the vortex ring. Thus, $R=\frac{\Gamma}{\mathrm{V}}$. Dahm et al. [1] have shown that the product $A F^{-2}$, for relatively small values of $A F^{-2}$ and in the absence of viscosity, governs the dynamics of the flow. (The limit $A F^{-2} \rightarrow 0$ defines the Boussinesq regime). Table 1 shows the parameter values for the numerical experiments presented in this extended abstract. Four calculations are shown, representing progressively weakening stratification.

Table 1. Numerical experiments

\begin{tabular}{|c|r|r|r|c|}
\hline & Run 1 & Run 2 & Run3 & Run4 \\
\hline $\mathrm{A}$ & $2 / 3$ & $2 / 3$ & $1 / 2$ & $1 / 3$ \\
\hline$F^{-2}$ & 0.5 & 0.0125 & 0.0125 & 0.6125 \\
\hline $\mathrm{R}$ & 5000 & 5000 & 5000 & 5000 \\
\hline Grid resolution & $250 \times 500$ & $250 \times 500$ & $200 \times 600$ & $150 \times 750$ \\
\hline
\end{tabular}

The full variable-density, incompressible Navier-Stokes equations in axisymmetric coordinates, together with the mass conservation equation, are solved:

$$
\begin{gathered}
U_{1}+(U \cdot \nabla) U=R^{-1} \Delta U-\frac{1}{\rho} \nabla p+F^{-2}\left(1-\frac{1}{\rho}\right) \\
\rho_{t}+(U \cdot \nabla) \rho=0 \\
\nabla \cdot U=0,
\end{gathered}
$$

where $U=\left[\begin{array}{l}u \\ v\end{array}\right]$ is the velocity vector, $\rho$ is the density, and $p$ is the pressure. 
In all of the calculations, the ring was started at a distance of $0.875 a$ below the interface, where $a$ is one ring diameter. The core was represented by a Gaussian vorticity profile with a ratio of core radius to ring radius of 0.25 . The interface was represented by a hyperbolic tangent profile with a transition thickness of $1 / 8$ the core diameter, well within the criteria for a "thin" interface. In all cases the fluid is stably stratified.

A video animation of these calculations has been made and will be shown when the paper is presented. Figures 1-4 are stills from that movie, and represent the corresponding Runs described in Table 1. It should be noted in interpreting these countour plots that, for purposes of visualization, the vorticity field was renormalized at each time step. The density field is shown at right; the vorticity field is reflected by symmetry into tie left half-plane.

In Run 1, the conditions are for a stiff interface. As the interface is approached by the vortex ring, it undergoes no visible defornation, and the vortex ring itself behaves as if is approaching a solid wall. It begins to expand, and a boundary layer is formed at the interface. The vortex peels counter-sign vorticity from the interface, and secondary and tertiary rings are formed which orbit around the primary ring. The counter-sign boundary layer vorticity ejects fluid back towards the symmetry axis. Eventually, a strong backflow is created, and the original ring is almost completely annhialated.

The conditions for Run 2 represent a slightly weaker interface (or stronger vortex ring). The interface deforms slightly with the approach of the ring, rising into a gentle mound and generating baroclinic vorticity along itsclf. Small pockets of light fluid, offset slightly to either side of the core of the ring, are scooped from above the interface and injected into the heavy fluid. The baroclinic vorticity is peeling counter-sign vorticity from the primary ring, reducing its strength and creating secondary and tertiary rings. The bubble collapses, a strong backflow jet is created which is Kelvin-Helmholtz unstable, and gravity waves propagate out along the interface.

Figures 3(a) and 3(b) show the interaction for an interface that is weaker still. Again, the interface deforms, and the baroclinic vorticity robs the primary vortex of much of its strength. The ring penetrates much further into the light fluid before collapsing, however, and the backflow jet appears to 
be dominated by a strong vortex ring of opposite sign (evolved from the interfacial vorticity) which entrains a large pocket of light fluid as it descends.

In Run 4, the interface is weakest; the path of the vortex ring is hardly affected at all. It travels through the interface and into the light fluid undisturbed. A nascent backflow jet is observed. These results show good qualitative agreement with the experiments and numerical simulations of Dahm et al [1].

Figures 5-8 show the $L^{\infty}$ (max) norm of the primary vorticity (initially associated with the ring) and the counter-sign vorticity (generated by interfacial effects) for Runs 1-4, respectively. The solid line indicates the primary vorticity; the counter-sign vorticity is represented by the dashed line. This not only provides a measure of the decay of the ring vortex and the growth of baroclinic and boundarylayer vorticity, it offers a striking visual representation of the degree of mixedness in the region affected by the interaction.

In Figure 5 (corresponding to Run 1), we see the rapid growth of a viscous boundary layer as the ring approaches the stiff interface. The large amplitude fluctuations in the counter-sign vorticity correspond to the ejection of secondary rings from the layer. These are clearly observed in Figure 1. The magnitudes of the positive and negative vorticity approach a common value, suggesting a wellmixed region.

For Run 2 (Figure 6), we observe a similar decay in the ring vorticity and rise in the counter-sign vorticity. It is difficult here to distinguish viscous effects from those due to baroclinicity, but again (as Figure 2 also indicates) the support of the vorticity is fairly well-mixed.

In Figures 7 and 8 , the decay in the primary vorticity is steeper, and the counter-sign vorticity production more gradual. The tendency towards large-amplitude fluctuations is reduced, and the curves appear increasingly independent, suggesting a weaker interaction and less mixing.

\section{Anticipated results}

In the final version of the paper, we will discuss the effects of variation in viscosity on the interaction, the symmetry-breaking characteristics of the non-Boussinesq regime, and the full threc- 
dimensional interaction, with particular emphasis placed on the phenomenon of vortex reconnection at the interface.

\section{References}

1. W. J. A. Dahm, C. M. Scheil, and G. Tryggvason, "Dynamics of vortex interaction with a density interface," J. Fluid Mech., vol. 205, pp. 1-43, 1989.

2. P. F. Linden, "The interaction of a vortex ring with a sharp density interface: a model for turbulent entrainment," J. Fluid Mech., vol. 60, pp. 467-480, 1973.

3. T. Sarpkaya, 16th Symposium on Naval Hydrodynamics (National Academy, Wash., D.C.), pp. 38-50, 1986.

4. W. W. Willmarth, G. Tryggvason, A. Hirsa, and D. Yu, Phys. Fluids A, vol. 1, p. 170, 1989.

5. D. L. Marcus and S.A. Berger, "The interaction between a counter-rotating vortex pair in vertical ascent and a frec surface," Phys. Fluids A, vol. 1 (12), pp. 1988-2000, 1989.

6. T. Sarpkaya, J. Elnitsky, II, and R. E. Lecker, Jr., 17th Symposium on Naval Hydrodynamics (National Academy, Wash., D.C.), pp. 53-60, 1989.

7. J. Telstc, J. Fluid Mech., vol. 201, p. 259, 1989.

8. J. B. Bell and D. L. Mareus, "A Second-Order Projection Method for Variable-Density Flows," Lawrence Livermore National Laboratory, Livermore, CA, UCRL-JC-104132(1990), submitted to J. Comp. Phys..

9. J. B. Bell, P. Colclla, and H. Glaz, "A Second-Order Projection Method for the Incompressible Navicr-Stokes Equations," J. Comp. Phys., pp. 257-283, December 1989.

10. A. J. Chorin, "On the Convergence of Discrete Approximations to the Navier-Stokes Equations," Math. Comp., vol. 23, pp. 341-353, April 1969.

11. A. B. Stephens, J. B. Bell, J. M. Solomon, and L. B. Hackerman, "A Finite Difference Galerkin Formulation of the Incompressible Navicr-Stokes Equations," J. Comp. Phys., vol. 53, pp, 152 - 
172, Jan. 1984.

12. J. M. Solomon aid W. G. Szymczak, "Finite Difference Solutions for the Incompressible Navier-Stokes Equations using Galerkin Techniques," Fifth IMACS International Symposium on Computer Methods for Partial Differential Equations, Lehigh University, June 19-21, 1984.

13. P. Colella, "Multidimensional Upwind Methods for Hyperbolic Conservation Laws," J. Comp. Phys., vol. 87, pp. 171-200, Mar. 1990. 


\section{List of Figures}

Figure 1. Vorticity and density contours; Run 1.

Figure 2. Vorticity and density contours; Run 2.

Figure 3(a)-(b). Vorticity and density contours; Run 3.

Figure 4. Vorticity and density contours; Run 4.

Figure 5. Magnitude of maximum vorticity vs, time. Solid line: primary (ring) vorticity, dashed line: counter-sign (interfacial) vorticity; Run 1.

Figure 6. Magnitude of maximum vorticity vs. time. Solid line: primary (ring) vorticity, dashed line: counter-sign (interfacial) vorticity; Run 2.

Figure 7. Magnitude of maximum vorticity vs. time. Solid line: primary (ring) vorticity, dashed line: counter-sign (interfacial) vorticity; Run 3.

Figure 8. Magnitude of maximum vorticity vs, time. Solid line: primary (ring) vorticity, dashed line: counter-sign (interfacial) vorticity; Run 4. 


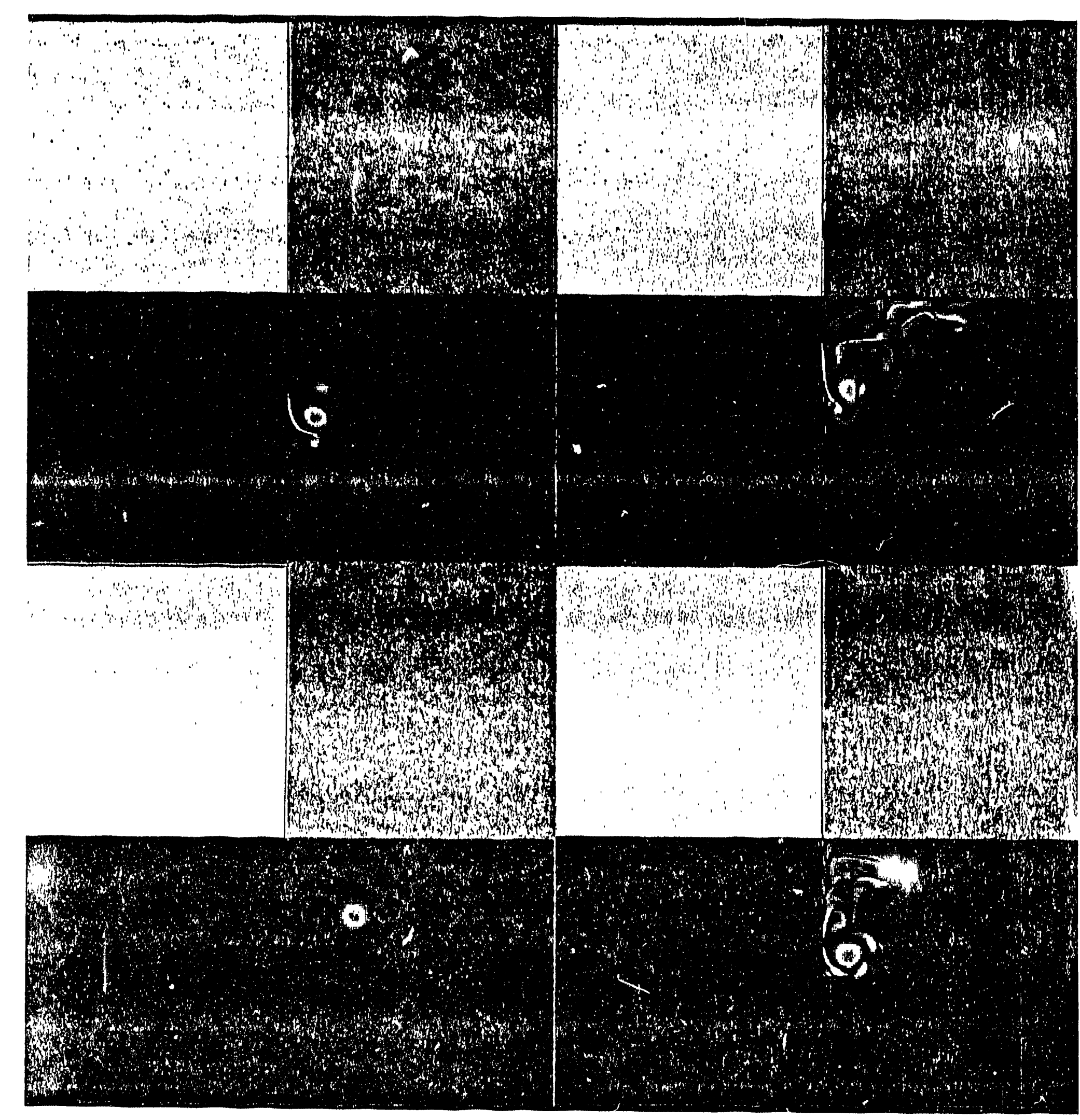



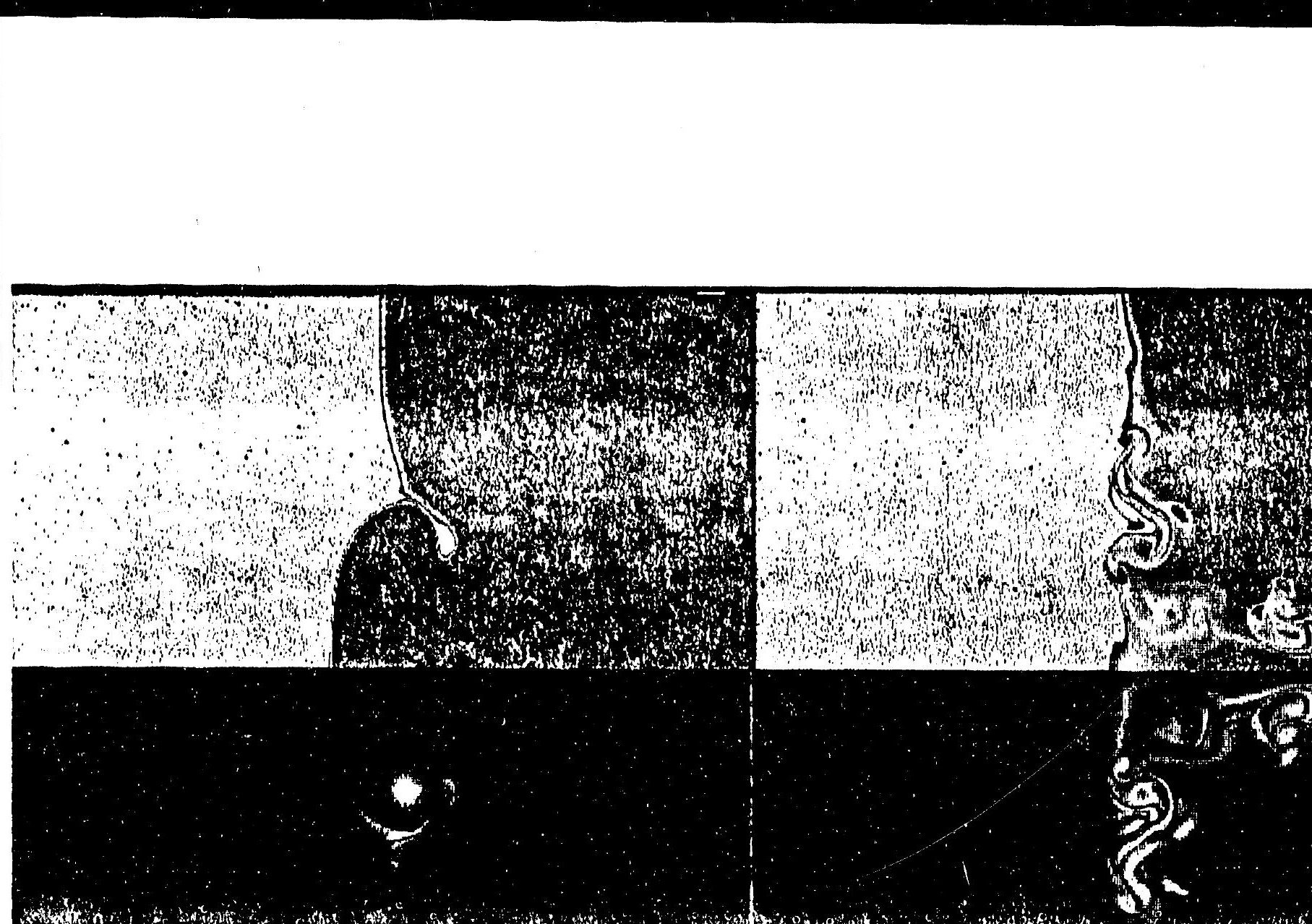

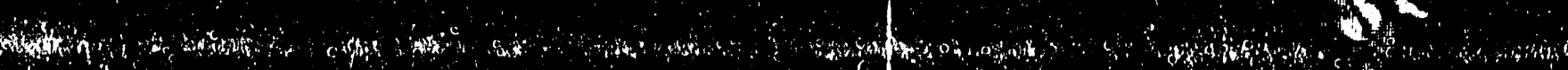

\section{$\Delta$}

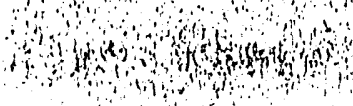

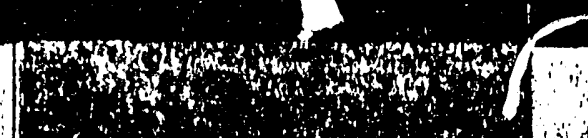

Chor
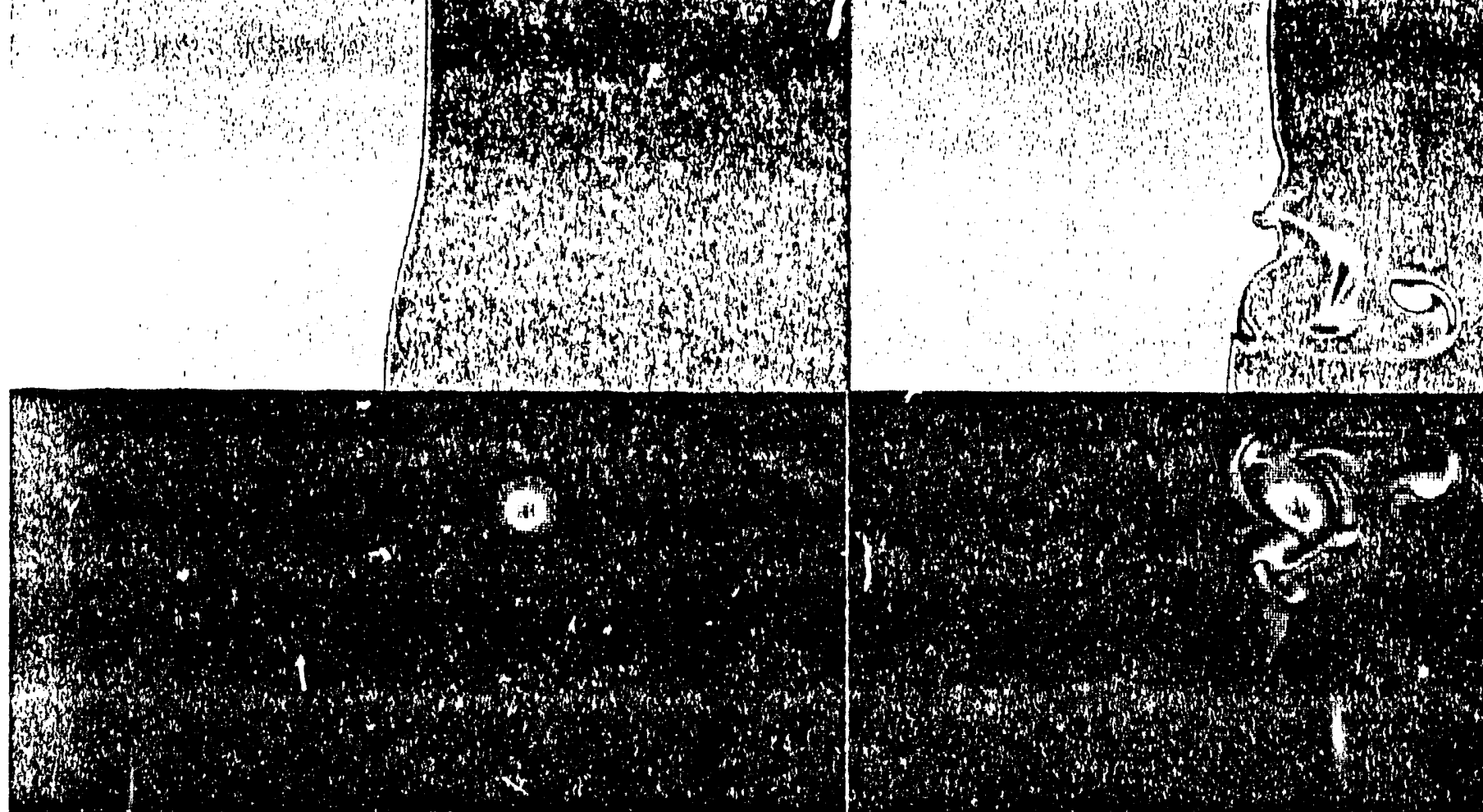
tiser. 


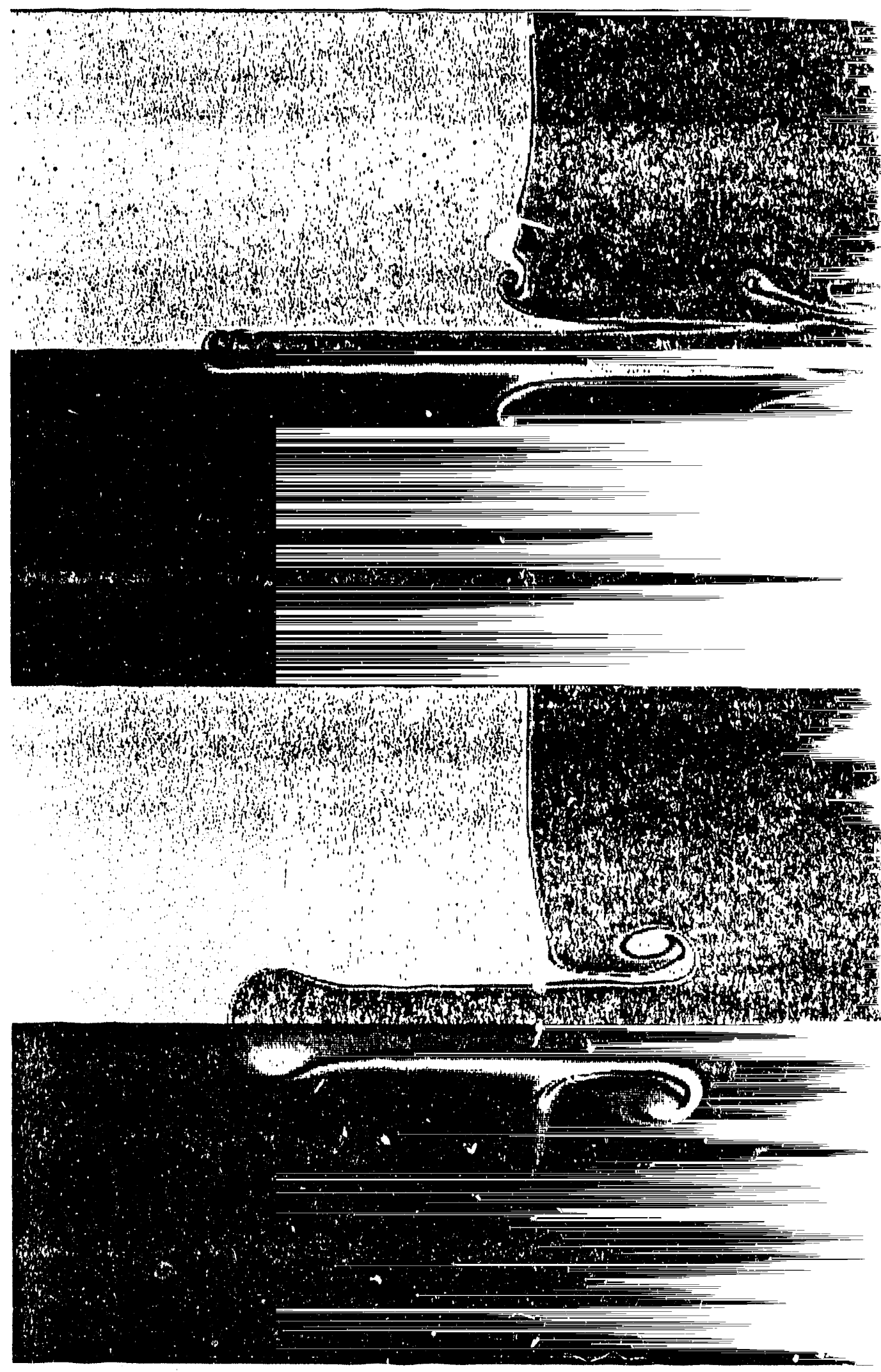




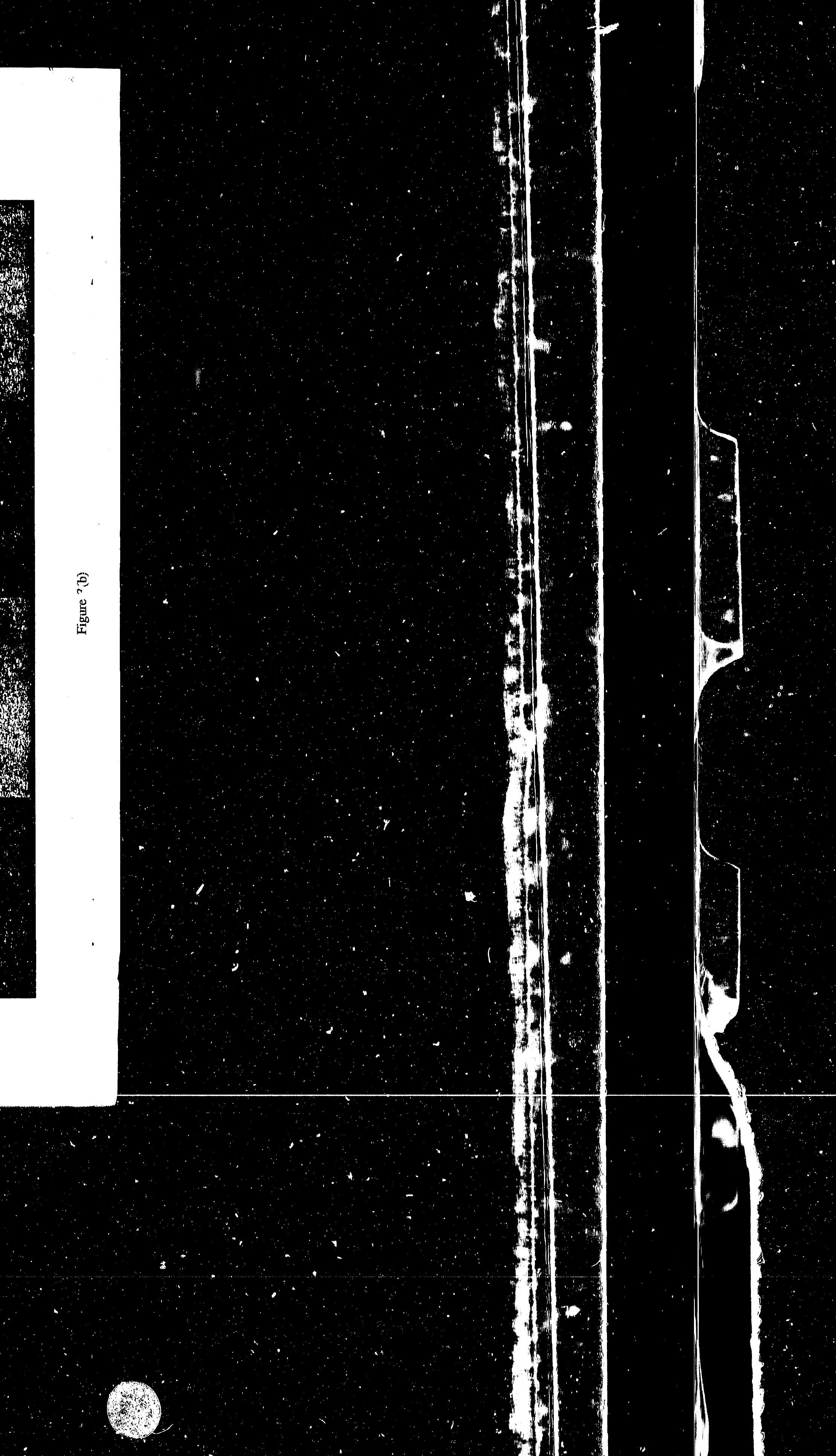




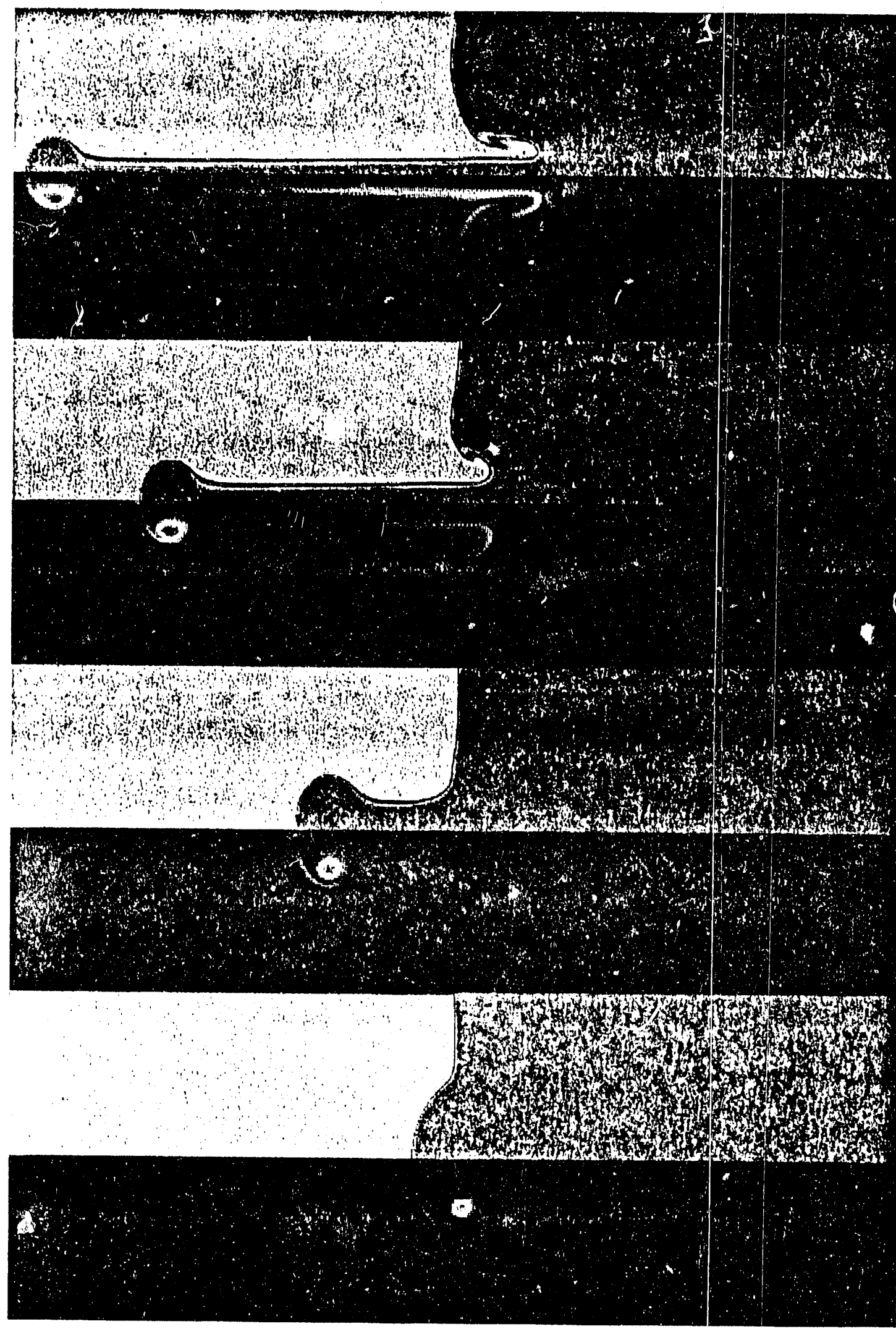




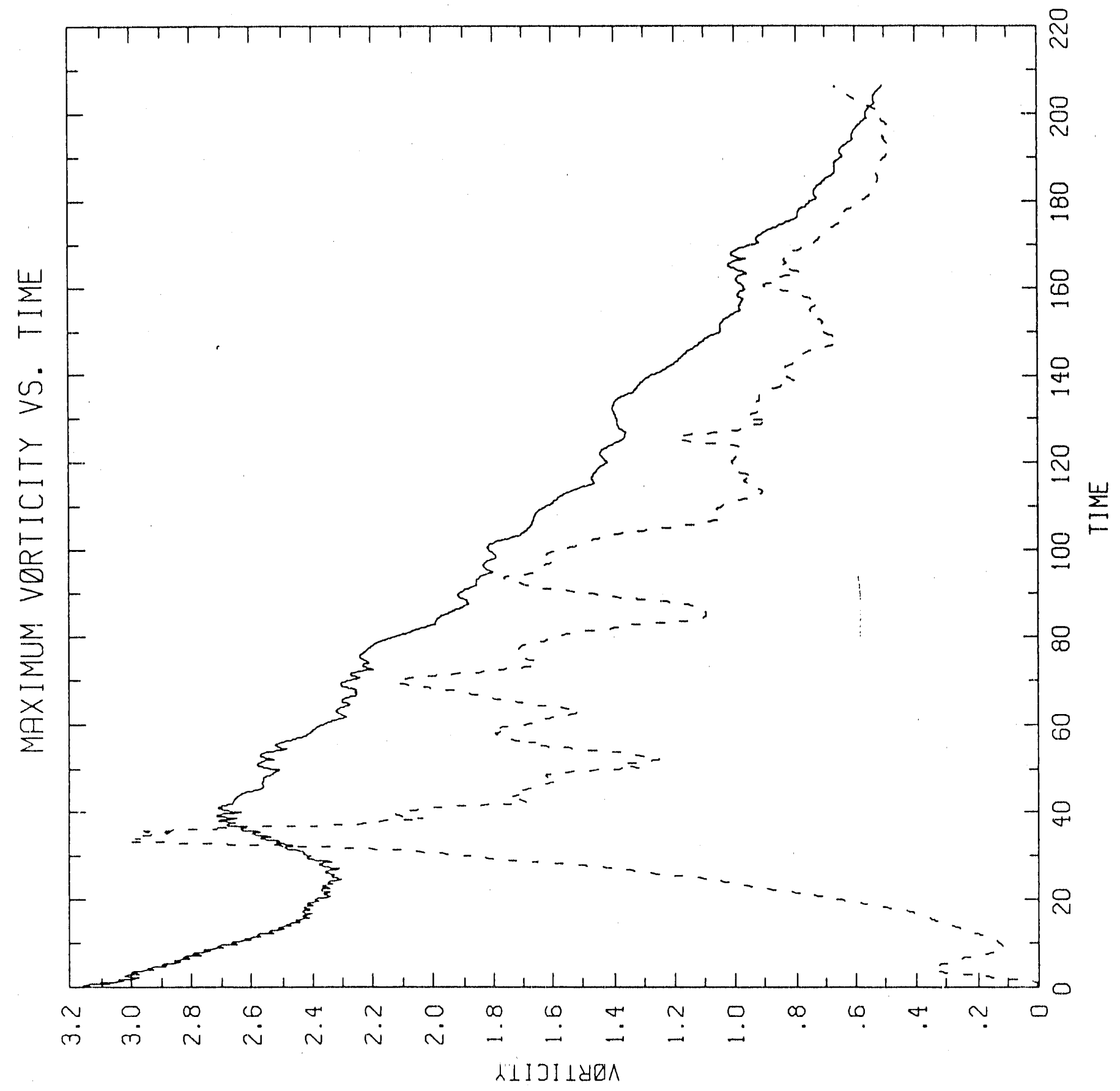




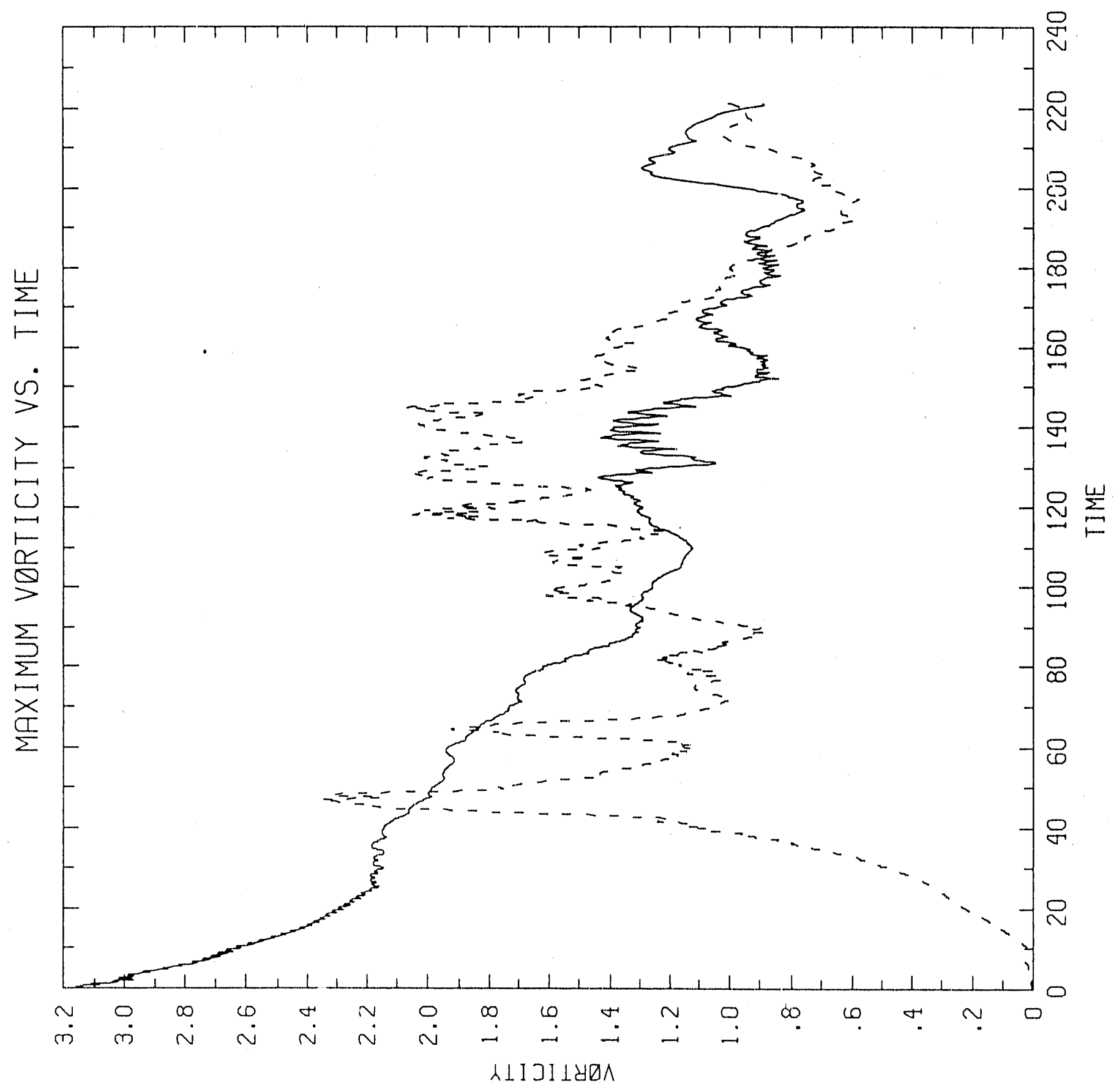




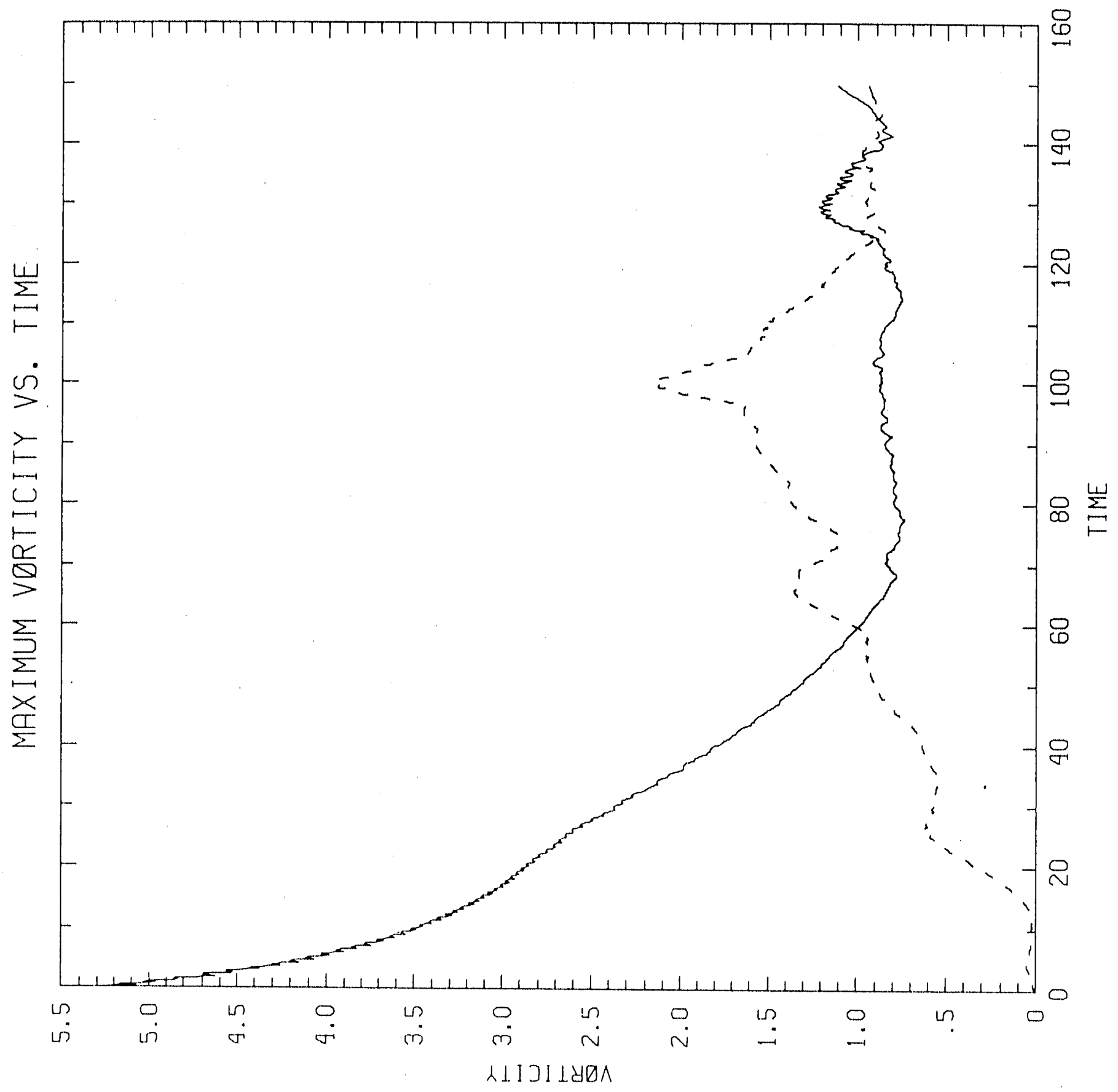




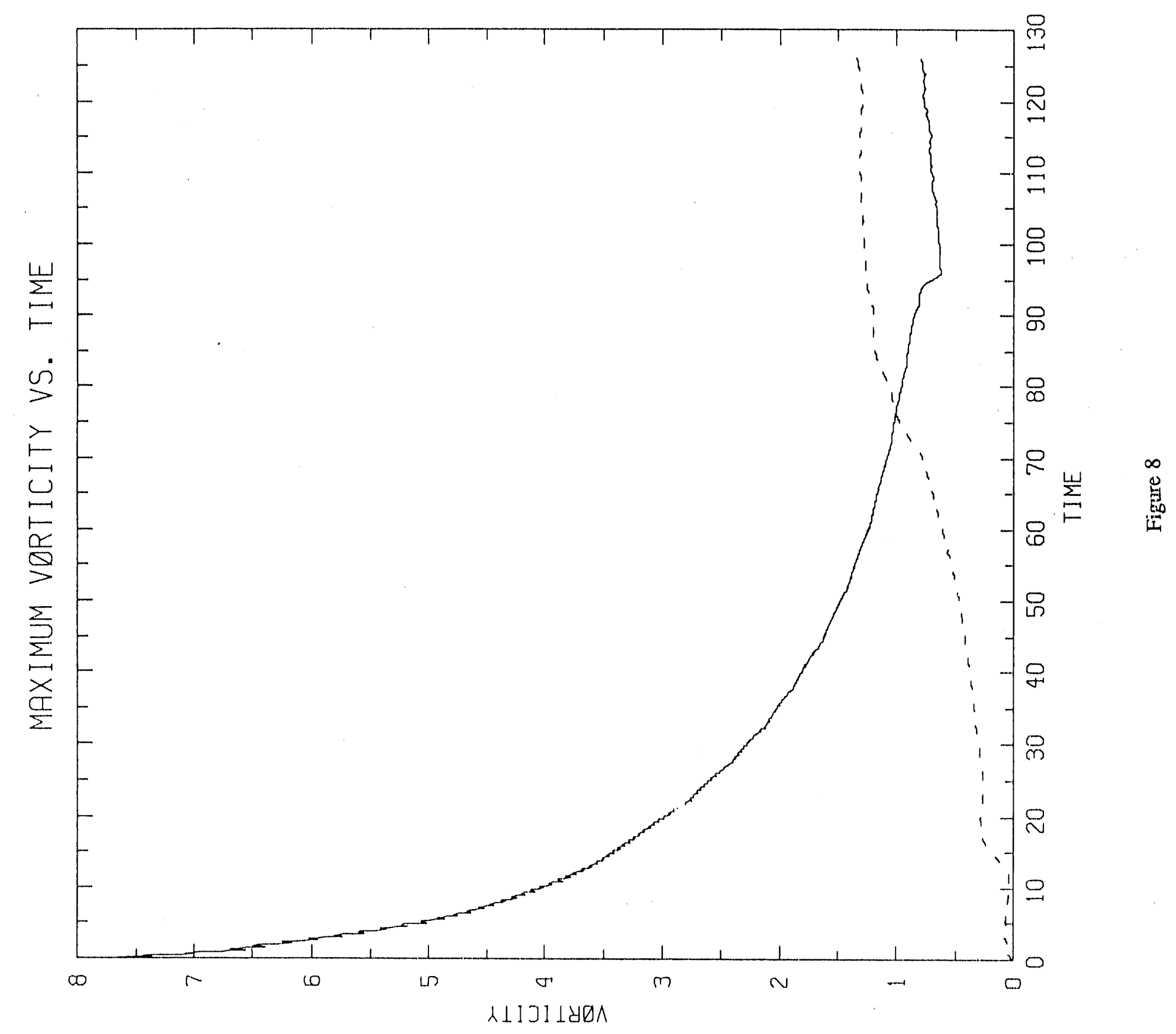



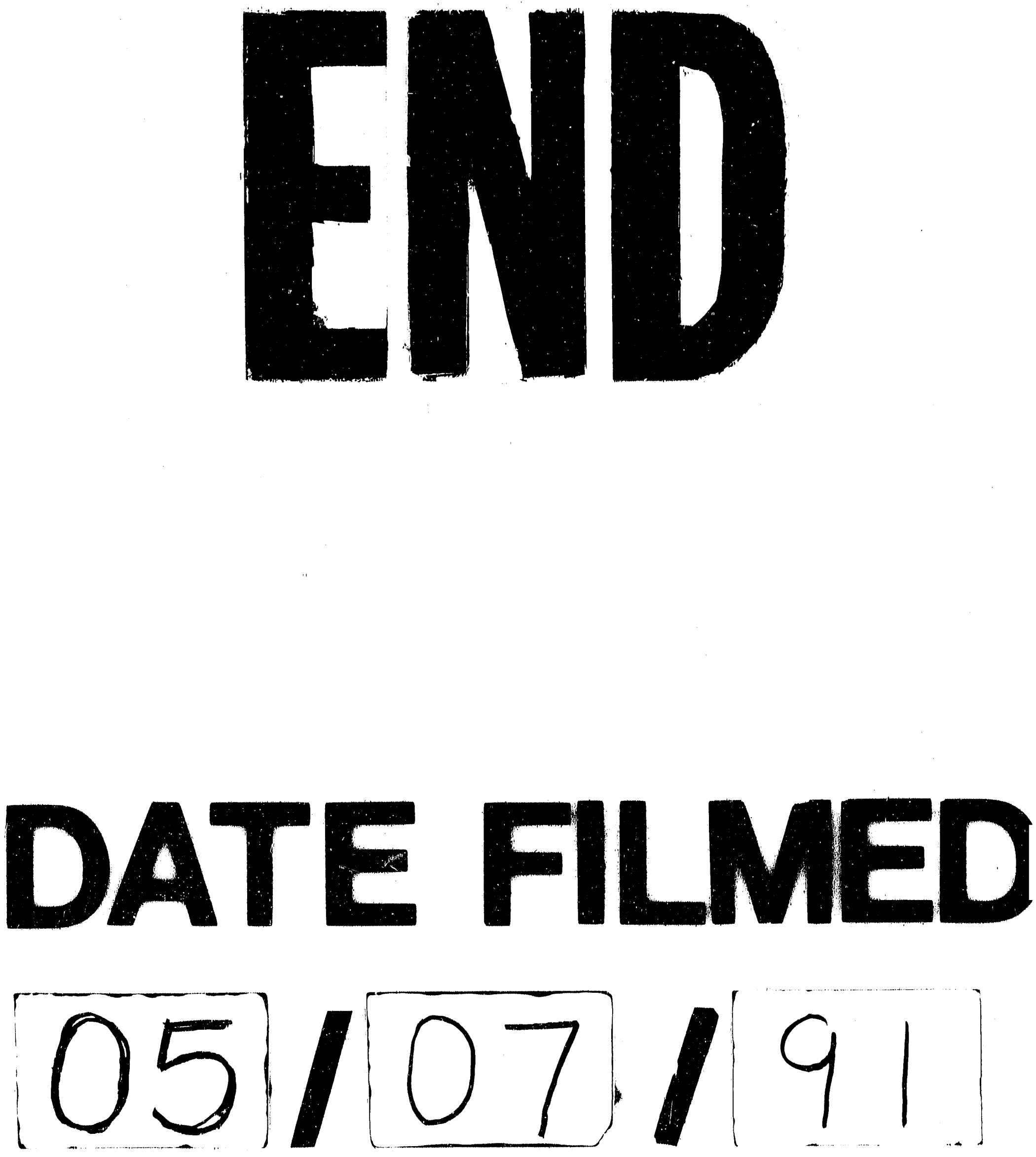
